Article

\title{
Evaluation of Age-Dependent Changes in the Coloration of Male Killifish Nothobranchius Guentheri Using New Photoprocessing Methods
}

\author{
Dmitry L. Nikiforov-Nikishin ${ }^{1,+} \mathbb{D}$, Nikita I. Kochetkov ${ }^{1, *},+\mathbb{D}$, Ekaterina V. Mikodina ${ }^{2}$,
} Alexei L. Nikiforov-Nikishin ${ }^{1}$, Yuri G. Simakov ${ }^{1}$, Natalya A. Golovacheva ${ }^{1}$, Alexander V. Gorbunov ${ }^{1}$, Sergei N. Chebotarev ${ }^{3}$, Evgeniya Yu. Kirichenko ${ }^{4}$, Igor Yu. Zabiyaka ${ }^{5}$, Ivan S. Pastukhov ${ }^{1}$ and Anzhelika B. Bren ${ }^{6}$

Citation: Nikiforov-Nikishin, D.L.; Kochetkov, N.I.; Mikodina, E.V.; Nikiforov-Nikishin, A.L.; Simakov, Y.G.; Golovacheva, N.A.; Gorbunov, A.V.; Chebotarev, S.N.; Kirichenko, E.Y.; Zabiyaka, I.Y.; et al. Evaluation of Age-Dependent Changes in the Coloration of Male Killifish Nothobranchius Guentheri Using New Photoprocessing Methods. Biology 2022, 11, 205. https:// doi.org/10.3390/biology11020205

Academic Editor: De-Li Shi

Received: 21 December 2021

Accepted: 26 January 2022

Published: 27 January 2022

Publisher's Note: MDPI stays neutral with regard to jurisdictional claims in published maps and institutional affiliations.

Copyright: (C) 2022 by the authors. Licensee MDPI, Basel, Switzerland. This article is an open access article distributed under the terms and conditions of the Creative Commons Attribution (CC BY) license (https:// creativecommons.org/licenses/by/ $4.0 /)$.
1 Department of Biotechnology and Fisheries, Moscow State University of Technologies and Management (FCU), 73, Zemlyanoy Val Str., 109004 Moscow, Russia; niknikdl@rambler.ru (D.L.N.-N.); 9150699@mail.ru (A.L.N.-N.); usimakov@yandex.ru (Y.G.S.); molekula00@inbox.ru (N.A.G.); akvabiotex@rambler.ru (A.V.G.); ivanpeskar@gmail.com (I.S.P.)

2 Russian Federal Research Institute of Fisheries and Oceanography (VNIRO), Verkhnyaya Krasnoselskaya St., 17, 107140 Moscow, Russia; arizon99@rambler.ru

3 Management Department, Moscow State University of Technologies and Management (FCU), 73, Zemlyanoy Val Str., 109004 Moscow, Russia; Chebotarev.sergei@gmail.com

4 Faculty "Bioengineering and Veterinary Medicine", Don State Technical University, Russian Federation, 1 Gagarin Sq., 344000 Rostov-on-Don, Russia; kler.smo@gmail.com

5 Department of Physical and Applied Material Science, Don State Technical University, Russia Federation, 1 Gagarin Sq., 344000 Rostov-on-Don, Russia; zabiyakaigor@gmail.com

6 Academy of Biology and Biotechnology, Southern Federal University, 344090 Rostov-on-Don, Russia; brenanzhelika@yandex.ru

* Correspondence: samatrixs@gmail.com; Tel.: +7-89260382261

+ These authors contributed equally to this work.

Simple Summary: This paper proposes a new methodology for evaluating fish coloration, which allows us to identify differences in the intensity of coloration of specific areas of the body. Changes in fish coloration occur during growth and under the influence of environmental factors. Male fish belonging to the family Nothobranchius are characterized by extremely diverse coloration, depending on the age of the fish, environmental factors, and social hierarchical status. As the lifespan of this genus of fish is very short (12-14 months), studies on age-dependent changes are possible. In this study, we demonstrate correlations between the coloration of particular body zones of male Nothobranchius guentheri and age using new photofixation methods and image processing software. This methodology can be applied to other fish with unique coloration patterns, for example, family Cichlidae and order Cyprinodontiformes.

\footnotetext{
Abstract: Fish as model objects have found wide applications in biology and fundamental medicine and allow studies of behavioral and physiological responses to various environmental factors. Representatives of the genus Nothobranchius are one of the most convenient objects for such studies. Male fish belonging to the family Nothobranchiidae are characterized by extremely diverse coloration, which constantly changes, depending on the age of the fish, environmental factors, and social hierarchical status. These fish species are characterized by a short life cycle, which allows changes in coloration, an indicator of the ontogenesis stage, to be estimated. Existing methods of fish color assessments do not allow the intensity of coloration of particular body zones to be clearly differentiated. In the present study, we suggest a method of two-factor assessment of specific fish body zones using modified methods of photofixation and image processing software. We describe the protocol of the method and the results of its application to different-aged groups of male Nothobranchius guentheri. The coloration of selected areas (i.e., red spot on the gill cover (RSGC), black border on the caudal fin (BBCF), and white border on the dorsal fin (WBDF)) differed significantly according to the size and age of the fish $(p<0.05)$. The data obtained suggest that $N$. guentheri can be a model for studying aging by the intensity of body coloration in males.
} 
Keywords: coloration; Nothobranchius guentheri; age-dependent changes; live fish photography; visualization of morphological characteristics

\section{Introduction}

Fish as model objects have found wide applications in biology and fundamental medicine and allow studies of behavioral and physiological responses to various environmental factors. In particular, Zebrafish (Danio rerio) [1] and medaka (Oryzias latipes) [2] have become widely used as representative teleosts. However, this model organism is not suitable for studying the aging process or changes in coloration with age due to the longer life span. In fish, coloration features and patterns are manifested during the course of reaching sexual maturity. It is difficult to study colorations and their changes at various stages of ontogenesis due to species differences and different rates of maturation [3].

The family Nothobranchiidae comprises a large group of fish, which mainly inhabit shallow ephemeral pools in northern Africa. As a result, they have formed a unique mechanism of reproduction and development, where the individual stages of ontogenesis are very close to each other [4]. Nothobranchiidae are short-lived, relatively easy to husbandry, transgenetic methods and genome sequencing have been determined, and they are much cheaper to keep than laboratory mice [5]. As such, representatives of this family are a convenient object for studying the processes of evolution, development, and aging [6-10].

The diverse coloration of male fish belonging to the Nothobranchiidae family cannot be explained only by mechanisms of sexual selection but is most likely a consequence of diverse ecological conditions, isolation of individual populations [11,12], and heterogeneity of this group of species. The following species are most often used to study the aging process: Nothobranchius furzeri [13], N. rachovii [14], and N. guentheri [15]. Each of these species has advantages and disadvantages in terms of studying the aging process. Among these species, $N$. rachovii has the shortest life cycle (5-6 months) but has one of the longest embryonic development durations (8-9 months), which makes its cultivation in laboratory conditions difficult. In contrast, $N$. guentheri has a longer life span and short embryonic diapause, which means that synchronized cultures can be obtained for research [8]. Representatives of the genus Nothobranchius are characterized by a high diversity of karyotypes, which, perhaps, plays an important role in their adaptation to unstable environmental conditions [16].

The coloration of male fish is characterized by phenotypic variability, which can vary within a wide range, both within members of different populations and among fish of the same group [11]. Thus, fish belonging to this genus appear to possess a divergent karyotype [17].

Changes in morphometric parameters of the body coloration of the genus Nothobranchius can provide an objective picture of maturation and aging (Figure 1).

The genus Nothobranchius is characterized by apparent sexual dimorphism and dichromatism [18]. As males of this genus are usually much brighter colored than females $[19,20]$, they can be used for color assessments. Criteria other than color (e.g., fertility) are used to assess growth-related parameters and maturation of female fish [21]. The presence of distinct differences in coloration and body shape suggests that visual signals play an important role in the choice of a sexual partner [18,22].

In addition to age, fish coloration, particularly coloration of the genus Nothobranchius, can change under the influence of the following factors: in response to environmental factors [23-25], social position in a group of individuals [26,27], feeding regime and food availability, drags affecting the neurohumoral system (e.g., exogenous androgens) [20,28], and according to some authors (N. Papa, personal communication [29]), during the aging process. 


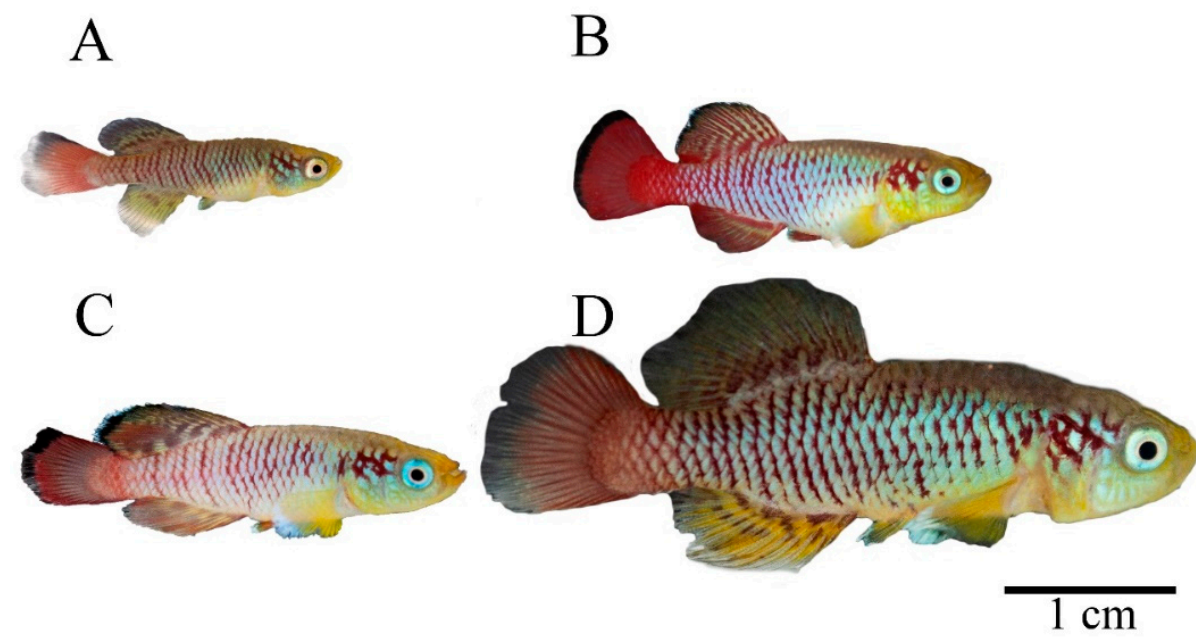

Figure 1. Morphological changes in the coloration of male N. guentheri, depending on hierarchical status and age. (A) Young male with signs of primary coloration (1.5 months); (B) Young male of high hierarchical status (4.5 months); (C) Adult male (6 months); (D) Adult male with signs of coloration regression (8 months).

The primary aim of the present study was to investigate coloration changes in $N$. guentheri males, depending on the age and size of the fish. An additional aim was to develop a method of their quantitative measurement based on novel photofixation/photoprocessing methods, applicable to most species of the family Nothobranchiidae and other fish, which are characterized by unique coloration traits. Such a method will expand the use of $\mathrm{N}$. guentheri as an alternative model for biological studies. The hypothesis of the present study was that differences in the coloration of particular body zones of $N$. guentheri males would be associated with different stages of maturation and aging. This hypothesis is based on the assumption that due to the short life cycle in the ontogenesis of this fish species, there is a clear staging of age-related changes, including coloration.

\section{Materials and Methods}

\subsection{Fish Housing}

The isolate N. guentheri Zanzibar TAN 14-02 was obtained from the collection of the Engelhardt Institute of Molecular Biology of the Russian Academy of Sciences. In total, 400 individuals of both sexes (age range: $2-8$ months) were included in this study. The fish were divided on the basis of sex and size parameters and kept in $50 \mathrm{~L}$ aquarium tanks $\left(45 \times 45 \times 15 \mathrm{~cm}^{3}, \mathrm{~W} \times \mathrm{L} \times \mathrm{H}\right)$, with constant aeration at a temperature of $22 \pm 2{ }^{\circ} \mathrm{C}$ and $\mathrm{pH}$ of 7.2-7.6. The water in these holding tanks was changed according to the following schedule: partial (once every 2 days) and complete (once every 7 days). The fish were fed live food (Daphnia magna, larvae of the family Chironomidae, Artemia salina) twice a day, at 12:00 and 18:00 $\mathrm{h}$.

\subsection{Anesthesia of Fish}

For photofixation, the fish were anesthetized to ensure no locomotor activity during the imaging period (4-5 $\mathrm{min})$. In order to avoid stress, which induces changes in coloration [30], the fish were sedated in a solution of MS-222 (0.1 mg/L) [13], after which their physical activity significantly decreased $[31,32]$. Subsequently, the fish remained in the aquarium for 5 min.

After photofixation, each fish was moved to a preprepared rehabilitation tank, where, after full recovery of cognitive function [33], the fish was returned to the holding tank. In order to exclude the possibility of behavioral changes in response to the sedation procedure, the fish was not exposed to the photofixation procedure again until 5 days after the first session. 


\subsection{Imaging}

In digital imaging, there were two key goals: to ensure the reliability of the color display and to ensure the repeatability of the data obtained. Previous studies provided detailed descriptions of the principles underlying fish photography [34,35]. Photofixation was conducted in accordance with the protocols outlined in the aforementioned studies, taking into account the purposes of the present research and features of the coloring of the fish species in the present study.

An example of the imaging setup is shown in Figure S1b. The camera was installed in front of the aquarium tank at a distance sufficient for focusing. An artificial light source was used, taking care to ensure that the obtained camera image was clear, with no negative effects (glare, overlight, etc.). We used a studio diode ring illuminator (Raylab RL-0518 Kit; Raylab, St Petersburg, Russia), where the color temperature was set to $4200 \mathrm{~K}$ (neutral color range), and a Nikon D5000 camera (Minato, Tokyo, Japan).

An aquarium tank with the following dimensions was used for fish photofixation: $55 \times 35 \times 20 \mathrm{~mm}^{3}$ (Figure S1a). The tank was made of quartz glass to achieve high optical transparency. The position of the tank remained unchanged during the whole period of photofixation to obtain high-quality photos. In order to compensate for artifacts and simplify further processing of the obtained image, the aquarium was set against a black background.

\subsection{Photographic Imaging Conditions}

In order to study age-dependent changes in coloration, the fish were divided into two groups based on the known age of the fish from the time of their hatching. The first group comprised individuals with a body length of $2.28-2.60 \mathrm{~cm}(2.5$ months $)(n=24)$, and the second group consisted of individuals with a body length of $3.10-4.18 \mathrm{~cm}$ (8 months) $(n=36)$.

Photofixation of living objects to quantify coloration is a complex methodological task [36]. Photofixation protocols previously described by a number of researchers $[34,37,38]$ were modified based on the species-specific characteristics of N. guentheri. The study design is presented in Figure S3. In our experiments, to obtain photographs suitable for further processing, the criteria were as follows: the fish remained motionless during the whole imaging period (see Section 2.2), the left lateral part with all morphological features of the fish [26] was visible on the image, and for reliable coloration evaluation, all the photographs of the fish were taken under identical lighting conditions (4200 K) and using the same camera settings (ISO 400-500, F 5.6, 1/60 s). Due to the strict standardization of the imaging conditions, color balance image calibration, usually used in similar studies [34,39], was not required.

Photofixation was performed according to the following protocol: male N. guentheri fish were moved from the housing tank to the sedation tank in groups of 10 individuals. After the onset of sedation, each fish was moved to the aquarium tank for photofixation. After the photographs were obtained, the fish (not more than 2-3 min postsedation) was moved to a specially prepared container for rehabilitation. In total, 104 male N. guentheri of different ages were photographed. From the database of the obtained photographs, we selected 60 of the most representative images suitable for processing (images with defects and distortions were not used).

\subsection{Image Processing}

Digital images in RAW format were processed using Adobe Photoshop ${ }^{\circledR}$ software, using standard tools to crop the image of each fish from the original image. Image processing to obtain numerical data on coloration intensity was performed using ImageJ software (Wayne Rasband (NIH); https:/ /imagej.nih.gov/ij/; accessed on 21 August 2021). The image processing technique was as described in previous studies [30,39-41]. The colors were coded according to hue, saturation, and brightness parameters [42]. 
In order to obtain reliable numerical data on coloration intensity, we apply a new method where fish coloration is presented as a set of reference zones, which are then evaluated according to two criteria: the coloration area and the modal (MoGV) or mean (MeGV) gray value. The basis of this method is the way in which a digital photo is represented as a set of pixels, each of which has a corresponding value defined by a color model. Once the resolution of the photographic image is sufficient, digital data can be obtained for further processing and statistical analysis. Determining the color of a particular zone is directly dependent on the resolution of the photographic image, as a single pixel may not be included in the determined zone at a low resolution [36].

The grayscale point value is a color mode in which each component of the original image is assigned a value equal to a shade of gray (from 1 to 255). In this way, the lightest/whitest pixels are assigned values close to 255, whereas the darkest/blackest pixels areas assigned values closer to zero. Applying this mode of displaying the zones under study allows us to estimate the degree of coloring intensity.

For this study, the authors identified two sets of representative coloration zones that most clearly reflect the phenotypic diversity of N. guentheri males and also play an important role in determining the social rank of an individual. Brightly colored body areas (zones) predetermine sexual behavior and mate choice [43] and are primally located on the lateral side of fish. In other fish species, similar zones with similar functional significance can be distinguished $[43,44]$.

The first set of body zones are visually the most distinguishable and are as follows (Figure 2):

- $\quad$ Red spot on the gill cover (RSGC);

- $\quad$ Black border on the caudal fin (BBCF);

- White border on the dorsal fin (WBDF).

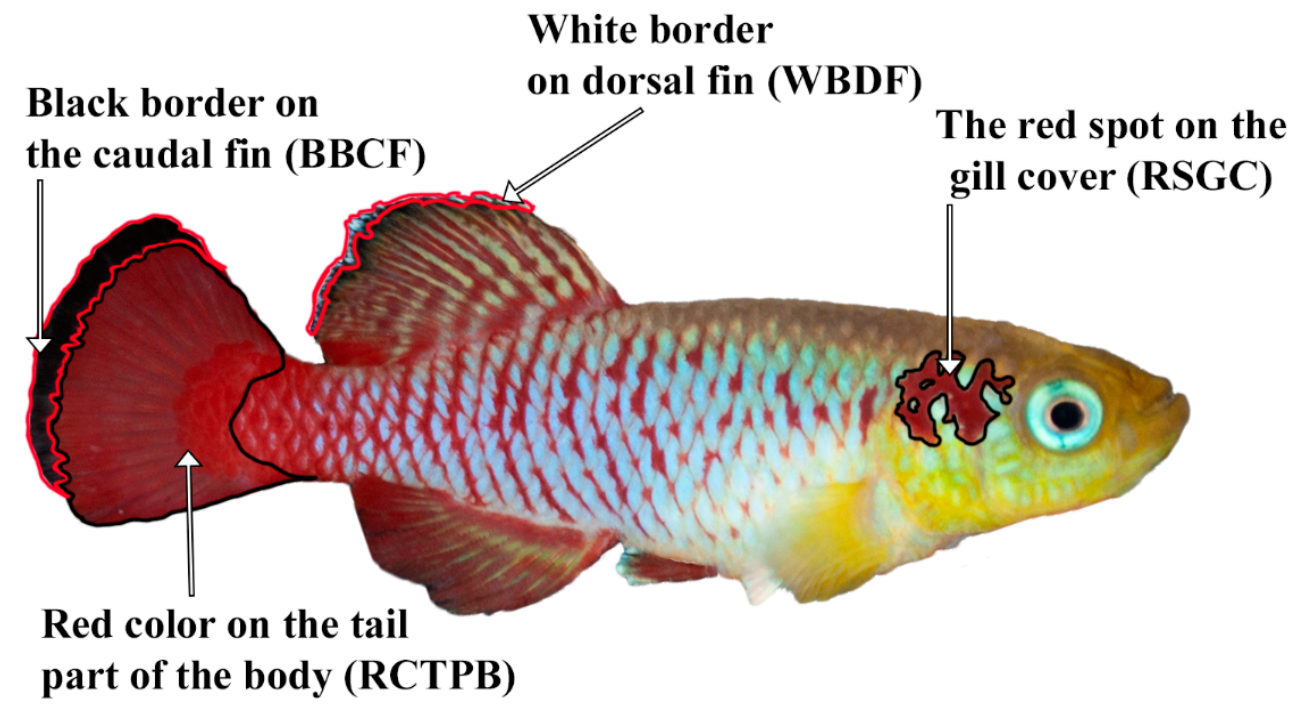

Figure 2. First set of body zones used in the measurement of N. guentheri.

The names of the first set of zones are based on the subjective perception of colors and used only to describe them in the text. The WBDF and RCTPB zones were not used in this study but may be used in further research

The zones in the first group are distinguished by the fact that they appear at the earliest stages of postembryonic development (3-4 weeks post-hatching) and remain visible, even in the presence of a marked change in fish coloration in response to external factors. In our opinion, this set of zones most accurately reflects the phenotypic dispersion within the studied isolate N. guentheri. Phenotypic dispersion should be minimal, as fish belong to the same isolate and has a similar genetic structure. 
The zones in the second group comprised a set of areas on the lateral part of the fish, matching the chosen gradation of colors. The zones used in the present study were:

- Zone of black zones on the dorsal and caudal fins, black region of interest (BROI) (Figure S2c);

- Zone of red zones on the entire lateral part, red region of interest (RROI) (Figure S2b);

- Zone of light blue areas on the entire lateral part, light blue region of interest (LBROI) (Figure S2a).

These zones were selected using the threshold color tool in ImageJ, adjusting the hue, saturation, and brightness of the original image (Figure S2). Numerical processing and estimation of the values of the zones in the second group allowed us to obtain the maximum data (MoFV, MeGV) from discrete information. We believe the first and second sets of zones can be considered objective criteria to evaluate coloration intensity, as they accurately reflect coloration in $\mathrm{N}$. guentheri males linked to age and social status.

\subsection{Statistical Analysis}

The data are presented as mean \pm standard deviation (SD). A comparative analysis of the different study groups was performed using the Student's t-criterion. A $p$-value of $<0.05$ was accepted as statistically significant. In order to determine the normality of the data distribution, the Shapiro-Wilk test was used. The correlation between different morphometric parameters was determined using Pearson's correlation, with the Student's $\mathrm{t}$-distribution used to calculate the significance. Paired linear regression was performed for parameters with a significant value of the correlation coefficient. The correlation lines were compared using an analysis of covariance (ANCOVA). Statistical data processing was performed using GraphPad Prism version 8.0 software (GraphPad, San Diego, CA, USA).

\section{Results}

The graphs in Figure 3 and Table 1 show the measurement data for the 60 experimental fish by the studied zones and MoGV and MeGV parameters. Numerical data are presented in Tables 1 and 2. The angle of slope of the correlation line significantly differed from zero $(p<0.05)$ in the zones: MeGV for BROI $\left(R^{2}=0.1512\right)$; MoGV and MeGV for LBROI, respectively $\left(R^{2}=0.07053\right.$ and $\left.R^{2}=0.07935\right)$. The most significant correlation between fish size and the studied gray values was noted in the BROI zone.
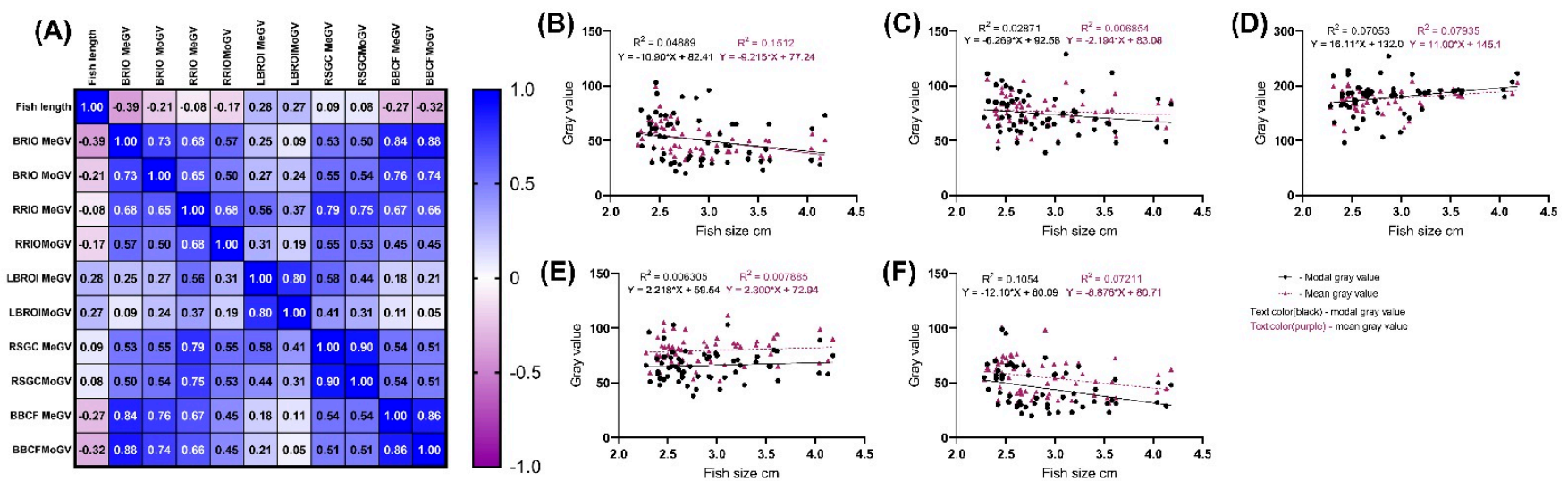

Figure 3. Linear regression of MoGV and MeGV parameters as a function of male N. guentheri size. (A) Person's correlation matrix between different zone. (B) BROI linear regression; (C) LBRIO linear regression; (D) RROI linear regression; (E) BBCF linear regression; (F) RSGC linear regression. 
Table 1. Numerical measurement data and the MoGVs for the two sets of zones in the entire sample. The value $(p<0.05)$ from unpaired $t$-test with Welch's correction.

\begin{tabular}{ccccc}
\hline & $\mathbf{8}$ MPH & $\mathbf{2 . 5} \mathbf{\text { MPH }}$ & $\boldsymbol{p}$-Value & Whole Sample \\
\hline Fish length & $2.47 \pm 0.09$ & $3.16 \pm 0.44$ & $\leq 0.0001$ & $2.89 \pm 0.49$ \\
\hline BROI MeGV & $45.44 \pm 9.42$ & $59.11 \pm 15.27$ & 0.0145 & $50.91 \pm 13.75$ \\
\hline RROI MeGV & $75.49 \pm 12.45$ & $78.62 \pm 13.84$ & 0.8733 & $76.74 \pm 13$ \\
\hline LBROI MeGV & $183 \pm 27.63$ & $171.9 \pm 20.66$ & 0.2528 & $176.9 \pm 19.16$ \\
\hline BBCF MeGV & $50.03 \pm 14.65$ & $62.62 \pm 15.77$ & 0.0153 & $55.06 \pm 16.22$ \\
\hline RSGC MeGV & $78.98 \pm 13.46$ & $80.49 \pm 11.72$ & 0.9978 & $79.58 \pm 12.71$ \\
\hline BROI MoGV & $46.06 \pm 21.06$ & $57.46 \pm 20.23$ & 0.9525 & $50.62 \pm 21.31$ \\
\hline RROI MoGV & $71.72 \pm 18$ & $78.58 \pm 17.96$ & 0.5817 & $74.47 \pm 18.15$ \\
\hline LBROI MoGV & $180.2 \pm 17.62$ & $172.5 \pm 32.45$ & 0.8143 & $178.5 \pm 29.76$ \\
\hline BBCF MoGV & $38.19 \pm 14.34$ & $55.50 \pm 18.92$ & 0.0003 & $45.12 \pm 18.29$ \\
\hline RSGC MoGV & $64.97 \pm 13.56$ & $67.42 \pm 14.07$ & 0.8563 & $65.95 \pm 13.70$ \\
\hline
\end{tabular}

Table 2. Numerical data on $X$ and the results of what analysis of the zones in the two groups. The value $(p<0.05)$ from unpaired $t$-test with Welch's correction.

\begin{tabular}{cccccc}
\hline & BROI Area & RROI Area & LBROI Area & RSGC Area & BBCF Area \\
\hline $8 \mathrm{MPH}$ & $52.95 \pm 24.26$ & $371.7 \pm 182$ & $385.1 \pm 155.1$ & $17.75 \pm 8.62$ & $36.38 \pm 28.44$ \\
\hline $2.5 \mathrm{MPH}$ & $44.67 \pm 23.9$ & $197.4 \pm 106.9$ & $205.5 \pm 59.21$ & $8.13 \pm 3.45$ & $27.85 \pm 11.42$ \\
\hline$p$-value & 0.1975 & $<0.0001$ & $\leq 0.001$ & $\leq 0.001$ & 0.1686 \\
\hline $\begin{array}{c}\text { Whole } \\
\text { sample }\end{array}$ & $49.64 \pm 24.46$ & $302 \pm 177.5$ & $313.3 \pm 153.3$ & $13.91 \pm 8.44$ & $32.97 \pm 23.42$ \\
\hline
\end{tabular}

For the first set of zones (BROI, LBROI, and RROI), the regression distributions of both studied gray values (MoGV and MeGV) coincided, and the slope angles did not differ significantly, resulting in a straight line. In the zones in the second group (RSGC and BBCF), we can discuss the reliable parallelism of the regression lines $(p<0.01)$ (Figure 3e,f).

In all the studied zones, the area was dependent on the size of the individual (Figure 4, Table 2). In all the studied zones, the angle of slope of the correlation line was significantly non-zero $(p<0.05)$. The smallest scatter interval and the highest correlation coefficient were noted in the following groups: RSGC $\left(R^{2}=0.7444\right)$ and LBROI $\left(R^{2}=0.723\right)$. For other coloration zones, the correlation coefficient was lower, with $R^{2}=0.05676$ for the BBCF zone.

In most cases, the selected areas in each of the two studied zones showed a clear correlation with the size of the fish. Coloration area increased in accordance with the age of the fish. A small proportion of fish due to phenotypic plasticity go beyond the range of the correlation line.

For a more adequate representation of the results of the study, the male fish were divided into two groups according to size: $2.51 \pm 0.11 \mathrm{~cm}$ ( 2.5 months old) and $3.26 \pm 0.42 \mathrm{~cm}$ (8 months old) (Figure S4).

As shown in Figure 5, in the zones in the first group, there was a significant difference only in the parameter BROI between the MeGV for both age groups of fish $(p<0.05)$. 


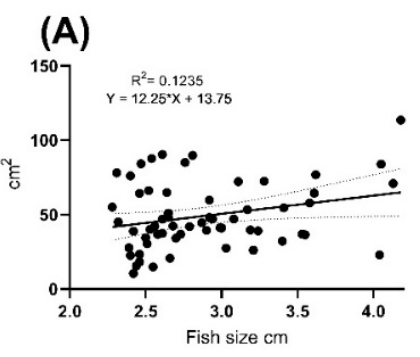

(D)

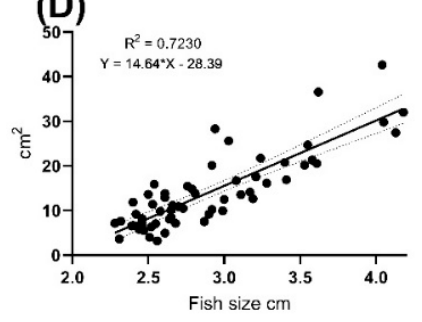

(B)

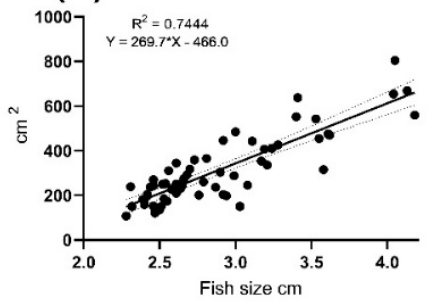

(C)

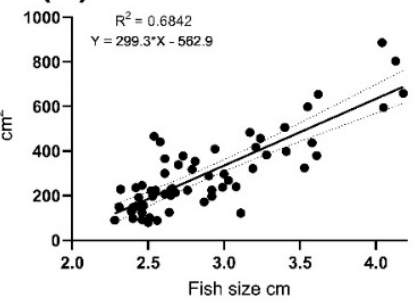

(E)

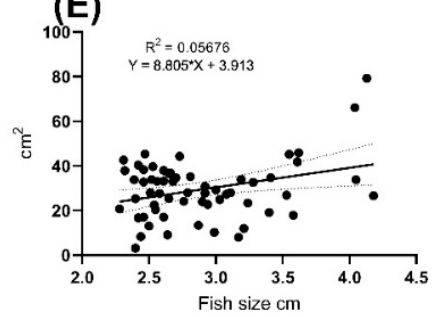

Figure 4. Linear regression of MoGV and MeGV parameters as a function of male N. guentheri size. (A) BROI linear regression; (B) LBRIO linear regression; (C) RROI linear regression; (D) BBCF linear regression; (E) RSGC linear regression.

(A)

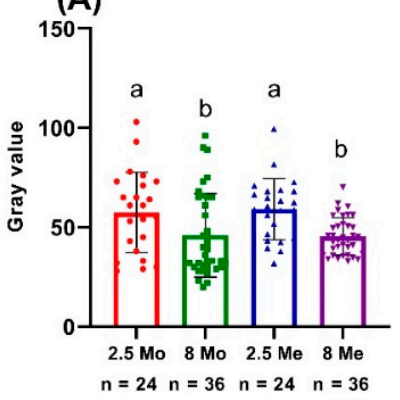

(D)
(B)

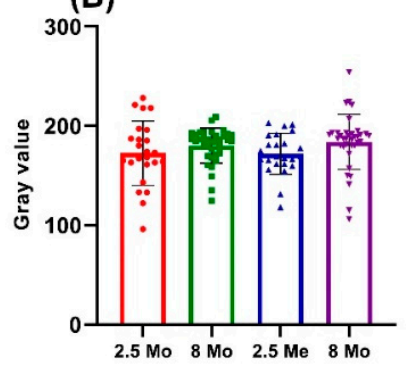

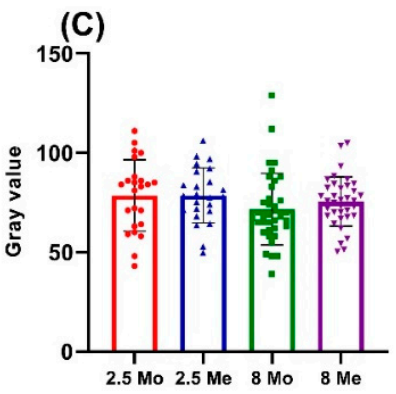
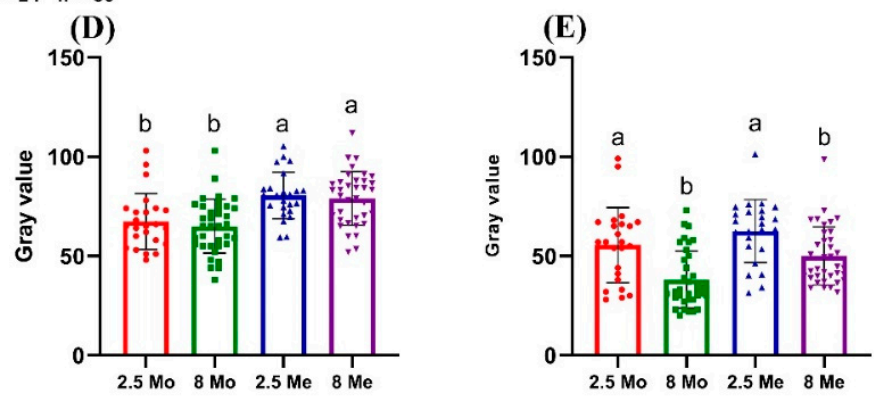

Figure 5. Comparison of MoGV and MeGV parameters in 2.5- and 8-month-old fish. The value $(p<0.05)$ from one-way ANOVA with comparison using Tukey's post hoc analysis. Different superscript letters $(a, b)$ indicate statistically significant differences between the experimental groups. (A) BROI zone; (B) LBRIO zone; (C) RROI zone; (D) RSGC zone; (E) BBCF zone.

In the RSGC zone, a significant difference was noted between the MoGVs and MeGVs of the different age groups $(p<0.001)$. In the BBCF zone, the MoGV of 2.5-month-old individuals differed significantly from that of 8-month-old individuals $(p<0.05)$, as well as in the MeGV $(p<0.001)$. In the two groups of zones, there was a significant difference in coloration intensity, as shown by the MoGVs and MeGVs $(p<0.001)$. For specific coloration areas, the MeGV value was a more reliable measurement parameter than the MoGV.

Comparison of the staining areas of the studied zones (Figure 6, Table 2) showed that the intensity of coloration of the following areas differed significantly in fish of different ages: LBRIO, RROI, and RSGC ( $p<0.05)$. 

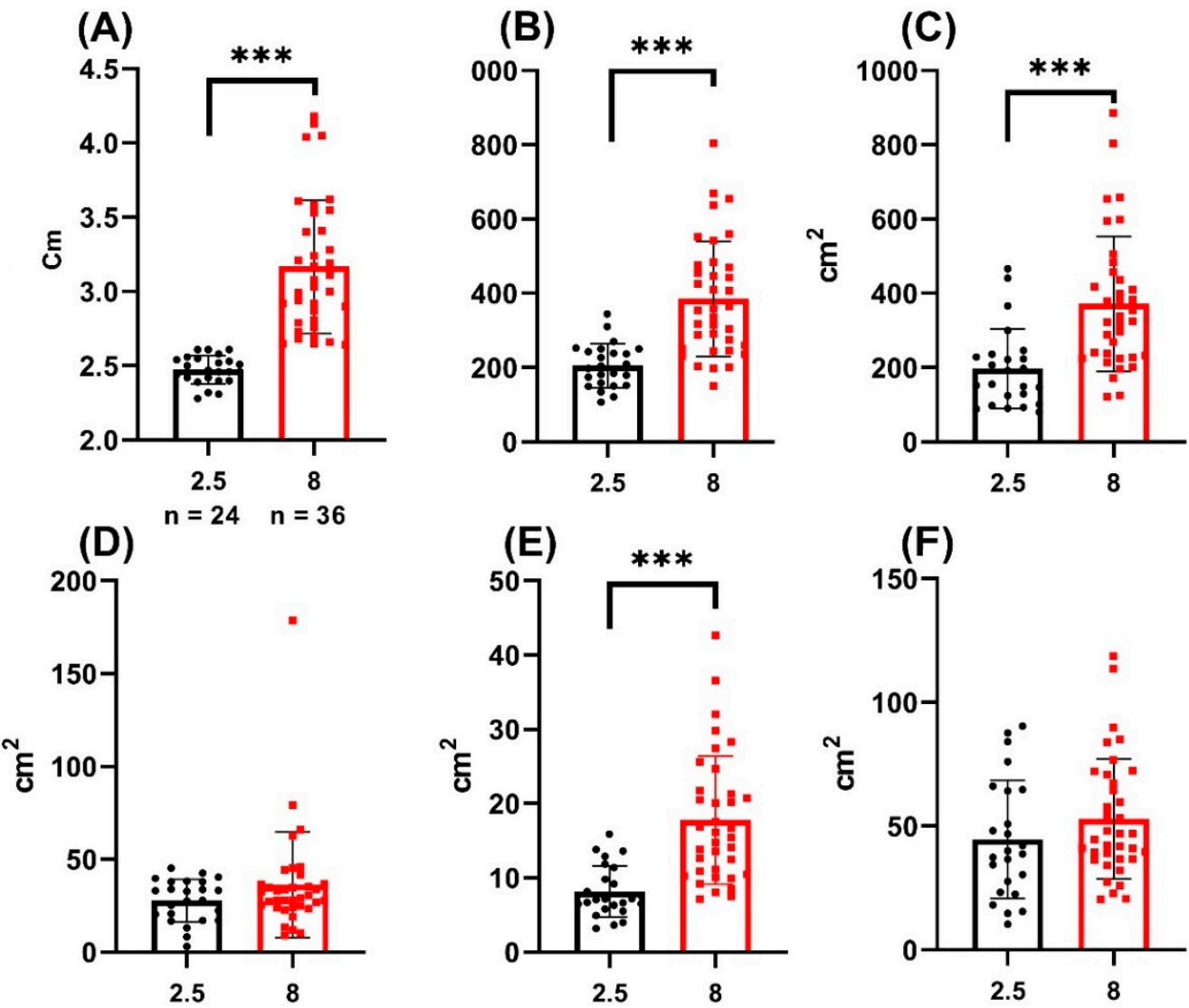

Figure 6. Comparison of the coloration areas of different zones of males of different age groups (2.5and 8-month post-hatching). The value $\left({ }^{* * *} p<0.001\right)$ from one-way ANOVA with comparison using Tukey's post hoc analysis. (A) fish length; (B) LBRIO zone; (C) RROI zone; (D) RSGC zone; (E) BROI zone; (F) BBCF zone.

\section{Discussion}

Previous methods designed to study fish coloration utilized the entire fish body and spectrocolorimetry [45]. Efforts to apply these methods to assess the coloration of male $N$. guentheri did not give positive results due to issues relating to laboriousness, the complexity of obtaining serial results, and the difficulty in measuring coloration parameters in fish with a complex morphological pattern of coloration. The methods proposed earlier are better suited for the estimation of large-sized fish with homogeneous coloration [46]. In our opinion, the methods proposed in this study for evaluating the coloration of male $N$. guentheri have advantages as compared to evaluation methods used previously $[34,35,37]$.

The development of digital photography makes it possible to obtain high-quality images, where each color zone corresponds to a large number of pixels [36]. Thus, general age- and size-related parameters, as well as the coloration of individual zones, can be assessed and compared, as demonstrated in this study.

Male fish, including male N. guentheri, can change the intensity of coloration depending on age, housing conditions, behavioral characteristics, and size [30]. The methodical approach proposed in this study makes it possible to standardize the color comparison in male N. guentheri fish.

In the zones in the first group, in the entire sample of individuals of different ages, the observed directions of linear regression can be explained by age-related changes in coloration, with brightening or darkening of the studied zones. The direction of the gray line correlation in different zones may be different (positive/negative).

Most likely, such variations in coloration are related to changes in the number of chromotocytes or pigment-protein content [47]. Changes in melanin content with age are 
characteristic of much bony fish, including N. guentheri [6]. As melanin is not the only pigment [48] involved in the formation of the coloration of the measured areas, the total value of gray varied within a slight range. Thus, in the BROI zone, age-related color brightening was clearly observed. The straight regression line for both MeGVs and MoGVs showed a negative direction. In turn, the LBROI zone had a positive regression, which points to the darkening of these zones with age. The multidirectional direction (positive in LBROI; negative BROI, BBCF) of the regression confirms the course of changes in fish coloration with increasing size and age, with these changes commencing at the time of sexual maturation and persisting almost until death $[49,50]$. Age-related variability can also be observed in fish with less bright coloration, although the variability is less distinct.

Some individuals differ significantly from the nominal coloration as a result of the phenotypical dispersion. The coloration of these fish cannot be interpreted within the framework applied in the present study. For example, Figure 7 shows a male N. guentheri, the coloration of which does not correspond to the coloration of individuals of his age and size. It can be assumed that the uncharacteristic coloration of this isolate is associated with intersex, which is observed in a percentage of individuals belonging to this family [51,52].

A

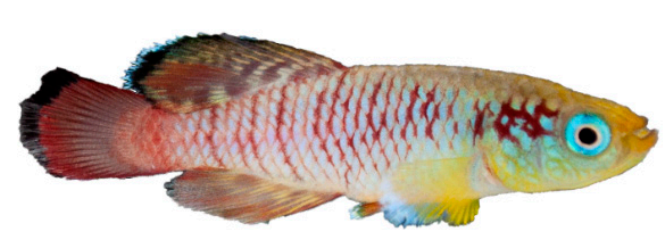

\begin{tabular}{|lrr|} 
& Modal GV & Mean GV \\
\hline BROI & 75 & 82.4 \\
\hline LBROI & 221 & 205.3 \\
RROI & 83 & 92.67 \\
RSGC & 75 & 84.71 \\
\hline BBCF & 75 & 78.28
\end{tabular}

B

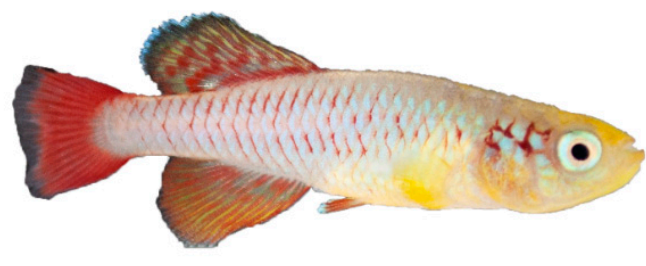

Figure 7. A comparison of the coloration of various male N. guentheri of comparable ages and sizes according to the gray value of the studied zones. (A) N. guentheri male with typical coloration and size. (B) Male with deviant type of coloration.

In the case of the zones in the first group, the differences between the MoGVs and MeGVs were minimal, as these zones of the fish body are characterized by uniform coloration, without pronounced individual differences. The stability of the coloration of these body zones may be a consequence of characteristic species or population traits that contribute to the recognition of fish in the group [53] and the choice of a mate [43,54]. The results of the present study are in accordance with those of previous research on coloration in the Cichlidae family [40].

The zones in the second group were characterized by a smaller coloration area. The results of the measurements of color intensity and those of the statistical analysis differed significantly in the two groups. Thus, the regression lines for the MoGVs and MeGVs did not overlap and were reliably parallel, and the mean values in both zones were almost always greater than the modal values. This may indicate that at the tissue level, pigment cells are located irregularly, causing diffuse changes in coloration [55,56]. As reported previously, pigment cells are arranged in layers, with xanthophores in the outer layer, iridophores in the middle layer, and melanophores in the inner skin layer $[57,58]$.

The slope angle of the regression line of the RSGC zone did not differ from zero, with the gray values remaining the same during the studied lifespan of the fish. The RSGC zone is an important phenotypic trait characteristic both for this isolate and for the species as a whole. It can be assumed that this zone plays an important role in the individual identification of fish and the determination of its hierarchical rank [43,53]. All red coloration 
zones can play a determining role in defining a partner's attractiveness $[59,60]$. In other fish species, in addition to red, orange and yellow often occur and perform the same function [61] but make the fish more visible to predators [62,63]. Therefore, the intensity of the coloration of the red zones is maintained at the same level regardless of size and age.

The results of the linear regression revealed a negative association between the BBCF zone and fish size and age, with the zone becoming darker. In N. guentheri male with typical coloration and size, the BBCF is black, which points to the presence of only one pigment, melanin, the content of which increases with age (Figure S4a,b). Black areas of coloration on the fish body may also have important evolutionary relevance for protection against solar insolation and the amount of incoming ultraviolet light, the effects of which can be deleterious in shallow ephemeral pools [64].

The comparison of the two different age groups of individuals according to the studied parameters showed results similar to those of the linear regression analysis. In the first group of zones, no significant differences were observed between the age groups in terms of MoGVs and MeGVs, except in the BRIO zone, where the MeGVs between old and young individuals differed significantly. This suggests that the MeGV is preferable to the MoGV for detecting differences in coloration.

In turn, the zones of the second group showed more significant results. Thus, both studied zones (RSGC and BBCF) showed significant differences between old and young individuals in MoGV and MeGV parameters. Given the reliability of both parameters in determining coloration intensity, one parameter is not superior to the other in calculating gray values.

Overall, the results of the color assessment method used in this study were more reliable when comparing coloration intensity in two different age groups of individuals than when comparing mass samples. As a result, there was a gradual decrease in the level of coloration intensity to minimal values in the group of older males. As this study did not include individuals with visible morphological changes as a result of aging, no dramatic changes in coloration were observed.

In this work, the intensity of coloration zones determined by phenotypic mechanisms was measured, but some aspects of coloration changes observed in social interaction (e.g., establishing social hierarchy) and group content may not have been observed. Some fishes show extremely short-term changes in coloration as a result of group interaction between males and changes in their hierarchical status $[65,66]$. It was noted that in the genus Nothobranchius, the change in coloration intensity in response to individual status is slow, as it is mediated hormonally rather than neuronally [67].

Changes in the patterns of coloration of male N. guentheri with aging are associated with complex physiological and biochemical reactions related to hormonal changes and the level of expression of genes responsible for the differentiation of pigment cells $[68,69]$. Age-dependent metabolic disorders cause regressive changes in the balance of apoptosis and blastomelanophore differentiation [69].

Despite the diversity of coloration of the different body regions of adult male $N$. guentheri, we were able to demonstrate significant changes in the intensity of coloration of individual areas according to the age and size of the fish using new photofixation methods and image processing software. The methods developed in this study can be applied to both short-lived representatives of the family Cyprinodontiformes and to other model organisms. It can be assumed that for fish with more uniform coloration, the measurements of coloration intensity will be easier due to the reduction in the number of zones that need to be measured. In addition, this measurement method is suitable both for assessing individual properties of fish and for studying the coloration intensity of large groups of individuals, which may be required in toxicological studies, studies of the effect of pharmacological drugs, and studies of the expression of genes responsible for changes in coloration [70], microevolutionary processes of phenotype change [22] and male-male aggression acts to suppress reproduction physiologically [22,71-73]. 
Based on the data collected, the coloration of male $N$. guentheri is highly variable, even in genetically homogeneous individuals. As the methods used in the present study do not require high-tech, costly equipment, they can be recommended for use in studies aimed at estimating color changes, even minor changes, in fish species. As members of this fish species have a short duration of ontogenesis, coloration changes that occur during most of their life cycle were considered in the present study. As shown by our results, changes in the coloration of male N. guentheri during its life cycle were expressed by changes in both the color intensity and size of specific areas in particular zones.

\section{Conclusions}

As shown by our results, the methods proposed in this study are suitable for comparative analysis of the coloration of $N$. guentheri males, both in individual fish and among groups of fish of different ages and sizes. The assessment of changes in fish coloration revealed patterns of change in both the overall color intensity and coloration of individual zones. When the entire sample consisting of individuals of different sizes was assessed, the selected zones reliably distinguished between individuals of different age groups. As shown by the measurements of specific areas of the body in particular zones, there was a positive association between these measurements and fish age. When the fish were divided into two groups by age and size, in the zones in the first group, there was a significant difference in average gray values only in the BROI zone. The zones in the second group showed reliable differences when comparing the groups among themselves, as well as within the same group, depending on the method of measurement. Based on the results of this study, the measurement of gray modal and mean values can be applied to assess age-related changes in coloration of male $N$. guentheri and other small fish with pronounced individual coloration. The results confirm the possibility of $N$. guentheri as a model organism for studying the mechanisms of development and aging.

Supplementary Materials: The following are available online at https: / www.mdpi.com/article / 10.3390/biology11020205/s1: Figure S1: Equipment for photofixation of N. guentheri. a Aquarium tank; b Experimental installation of the equipment with the specified lighting parameters.; Figure S2: Selected zones of the second group with the following values of brightness and saturation: A LBROI, saturation from $0 \pm 10$ to $85 \pm 10$; brightness from $145 \pm 10$ to $246 \pm 9$. B RROI, saturation, $70 \pm 10$ to $145 \pm 5$; brightness, $70 \pm 5$ to $140 \pm 5$; C BROI, saturation, 0 to $55 \pm 5$; brightness, 0 to $75 \pm 5$. ${ }^{*}$ All brightness and saturation values ( 1 to 255 ) are applicable only to the photographs obtained in this study.; Figure S3: Study design. The main stages of conducting and processing the material.; Figure S4: Distribution of fish into groups according to size and age.

Author Contributions: Conceptualization, methodology, D.L.N.-N. and N.I.K.; software, A.V.G.; validation, formal analysis, E.V.M. and A.B.B.; investigation, A.V.G., E.Y.K., Y.G.S.; resources, data curation, I.Y.Z. and S.N.C.; writing-original draft preparation, D.L.N.-N., N.I.K. and I.S.P.; writingreview and editing, project administration, A.L.N.-N.; visualization, N.I.K. and N.A.G.; All authors have read and agreed to the published version of the manuscript.

Funding: The research was financially supported by the Ministry of Science and Higher Education of the Russian Federation within the framework of the state task in the field of scientific activity (Southern Federal University, no. 0852-2020-0029).

Institutional Review Board Statement: The study was conducted according to the guidelines of the Declaration of Helsinki, and approved by the Institutional Ethics Committee of Razumovskogo Moscow State University of Technology and Management (approval number 8, 02/02/2021).

Informed Consent Statement: Not applicable.

Data Availability Statement: Not applicable.

Conflicts of Interest: The authors declare no conflict of interest. 


\section{References}

1. Rahman Khan, F.; Sulaiman Alhewairini, S. Zebrafish (Danio rerio) as a Model Organism. Curr. Trends Cancer Manag. 2019, 2019, 81517.

2. Naruse, K.; Sakaizumi, M.; Shima, A. Medaka as a model organism for research in experimental biology. Fish Biol. J. Medaka 1994, 6, 47-52.

3. Nüsslein-Volhard, C.; Singh, A.P. How fish color their skin: A paradigm for development and evolution of adult patterns: Multipotency, plasticity, and cell competition regulate proliferation and spreading of pigment cells in zebrafish coloration. BioEssays 2017, 39, 1600231. [CrossRef] [PubMed]

4. Furness, A.I. The evolution of an annual life cycle in killifish: Adaptation to ephemeral aquatic environments through embryonic diapause. Biol. Rev. 2016, 91, 796-812. [CrossRef] [PubMed]

5. Cellerino, C. Annual fishes of the genus Nothobranchius as an experimental model. Annu. Fishes Life Hist. Strategy Divers. Evol. 2016, 1, 93-98

6. Lucas-Sánchez, A.; Almaida-Pagán, P.F.; Mendiola, P.; de Costa, J. Nothobranchius as a model for aging studies. A review. Aging Dis. 2014, 5, 281-291. [CrossRef]

7. Herrera, M.; Jagadeeswaran, P. Annual fish as a genetic model for aging. J. Gerontol. Ser. A Biol. Sci. Med. Sci. 2004, 59, B101-B107. [CrossRef]

8. Nikiforov-Nikishin, D.L.; Irkha, V.A.; Kochetkov, N.I.; Kalita, T.L.; Nikiforov-Nikishin, A.L.; Blokhin, E.E.; Antipov, S.S.; Makarenkov, D.A.; Zhavnerov, A.N.; Glebova, I.A.; et al. Some Aspects of Development and Histological Structure of the Visual System of Nothobranchius Guentheri. Animals 2021, 11, 2755. [CrossRef]

9. Guvatova, Z.G.; Fedorova, M.S.; Vershinina, Y.S.; Pudova, E.A.; Lipatova, A.V.; Volodin, V.V.; Gladysh, N.; Tokarev, A.; Kornev, A.; Pavlov, V.; et al. De Novo Transcriptome Profiling of Brain Tissue from the Annual Killifish Nothobranchius guentheri. Life 2021, 11, 137. [CrossRef]

10. Aragona, M.; Porcino, C.; Guerrera, M.C.; Montalbano, G.; Levanti, M.; Abbate, F.; Laurà, R.; Germanà, A. Localization of Neurotrophin Specific Trk Receptors in Mechanosensory Systems of Killifish (Nothobranchius guentheri). Int. J. Mol. Sci. 2021, 22, 10411. [CrossRef]

11. Maan, M.E.; Sefc, K.M. Colour variation in cichlid fish: Developmental mechanisms, selective pressures and evolutionary consequences. Semin. Cell Dev. Biol. Acad. Press 2013, 24, 516-528. [CrossRef] [PubMed]

12. Sabaj, M.H.; Maurakis, E.G.; Woolcott, W.S. Spawning behaviors in the bluehead chub, Nocomis leptocephalus, river chub, N. micropogon and central stoneroller, Campostoma anomalum. Am. Midl. Nat. 2000, 144, 187-201. [CrossRef]

13. Hsu, C.Y.; Chiu, Y.-C.; Hsu, W.-L.; Chan, Y.-P. Age-related markers assayed at different developmental stages of the annual fish Nothobranchius rachovii. J. Gerontol. Ser. A Biol. Sci. Med. Sci. 2008, 63, 1267-1276. [CrossRef] [PubMed]

14. Valdesalici, S.; Cellerino, A. Extremely short lifespan in the annual fish Nothobranchius furzeri. Proc. R. Soc. Lond. Ser. B Biol. Sci. 2003, 270 (Suppl. S2), S189-S191. [CrossRef]

15. Haas, R. Sexual selection in Nothobranchius guentheri (Pisces: Cyprinodontidae). Evolution 1976, 30, 614-622. [CrossRef]

16. Krysanov, E.; Demidova, T. Extensive karyotype variability of African fish genus Nothobranchius (Cyprinodontiformes). Comp. Cytogenet. 2018, 12, 387-402. [CrossRef]

17. Krysanov, E.; Demidova, T.; Nagy, B. Divergent karyotypes of the annual killifish genus Nothobranchius (Cyprinodontiformes, Nothobranchiidae). Comp. Cytogenet. 2016, 10, 439. [CrossRef]

18. Passos, C.; Tassino, B.; Rosenthal, G.G.; Reichard, M. Reproductive Behavior and Sexual Selection in Annual Fishes; CRC Press: Boca Raton, FL, USA, 2015; pp. 207-230.

19. Yu, X.; Li, G. Effects of resveratrol on longevity, cognitive ability and aging-related histological markers in the annual fish Nothobranchius guentheri. Exp. Gerontol. 2012, 47, 940-949. [CrossRef]

20. Sedlácek, O.; Baciaková, B.; Kratochvíl, L. Evolution of body colouration in killifishes (Cyprinodontiformes: Aplocheilidae, Nothobranchiidae, Rivulidae): Is male ornamentation constrained by intersexual genetic correlation? Zool. Anz. J. Comp. Zool. 2014, 253, 207-215. [CrossRef]

21. Vrtílek, M.; Reichard, M. Highly plastic resource allocation to growth and reproduction in females of an A frican annual fish. Ecol. Freshw. Fish 2015, 24, 616-628. [CrossRef]

22. Reichard, M.; Polačik, M. Reproductive isolating barriers between colour-differentiated populations of an African annual killifish, Nothobranchius korthausae (Cyprinodontiformes). Biol. J. Linn. Soc. 2010, 100, 62-72. [CrossRef]

23. Proulx, R.; St-Laurent, M.H.; Parrott, L. Emergent phenotypes: Association between morphology and coloration in fish. Evol. Ecol. Res. 2008, 1, 1037-1050.

24. Alphen, J.J.M.V. Evolution of colour patterns in East African cichlid fish. J. Evol. Biol. 1999, 12, 514-534.

25. Reichard, M.; Polačik, M.; Sedláček, O. Distribution, colour polymorphism and habitat use of the African killifish Nothobranchius furzeri, the vertebrate with the shortest life span. J. Fish Biol. 2003, 74, 198-212. [CrossRef] [PubMed]

26. Dijkstra, P.D.; Maguire, S.M.; Harris, R.M.; Rodriguez, A.A.; DeAngelis, R.S.; Flores, S.A.; Hofmann, H.A. The melanocortin system regulates body pigmentation and social behaviour in a colour polymorphic cichlid fish. Proc. R. Soc. B Biol. Sci. 2017, 284, 20162838. [CrossRef] [PubMed]

27. Moretz, J.A.; Morris, M.R. Evolutionarily labile responses to a signal of aggressive intent. Proc. R. Soc. Lond. Ser. B Biol. Sci. 2003, 270, 2271-2277. [CrossRef] 
28. Kalinowski, C.T.; Robaina, L.; Fernández-Palacios, H.; Schuchardt, D.; Izquierdo, M. Effect of different carotenoid sources and their dietary levels on red porgy (Pagrus pagrus) growth and skin colour. Aquaculture 2005, 244, 223-231. [CrossRef]

29. de Sá, R.O.; Berois, N.; García, G. Overview, Future Challenges, and Evolution of Annualism. Annual Fishes: Life History Strategy, Diversity, and Evolution, 1st ed.; Press Taylor \& Francis Group: Boca Raton, FL, USA, 2015; pp. 309-318.

30. Kittilsen, S.; Schjolden, J.; Beitnes-Johansen, I.; Shaw, J.; Pottinger, T.; Sørensen, C.; Braastad, B.; Bakken, M.; Overli, O. Melaninbased skin spots reflect stress responsiveness in salmonid fish. Horm. Behav. 2009, 56, 292-298. [CrossRef]

31. Brown, L.A. Anesthesia in fish. Vet. Clin. N. Am. Small Anim. Pract. 1988, 18, 317-330. [CrossRef]

32. Neiffer, D.L.; Stamper, M.A. Fish sedation, anesthesia, analgesia, and euthanasia: Considerations, methods, and types of drugs. ILAR J. 2009, 50, 343-360. [CrossRef]

33. Chervova, L.S.; Lapshin, D.N. Behavioral control of the efficiency of pharmacological anesthesia in fish. J. Ichthyol. 2011, 51, 1126-1132. [CrossRef]

34. García-Melo, J.E.; García-Melo, L.J.; García-Melo, J.D.; Rojas-Briñez, D.K.; Guevara, G.; Maldonado-Ocampo, J.A. Photafish system: An affordable device for fish photography in the wild. Zootaxa 2019, 4554, 141-172. [CrossRef] [PubMed]

35. Lee, J.Y.; Kweon, H.S.; Park, E.H. Improved photographic technique for enhancing the morphological resolution of small juvenile fishes. Ichthyol. Res. 1999, 46, 423-425. [CrossRef]

36. Stevens, M.; Kweon, H.-S.; Park, E.-H. Using digital photography to study animal coloration. Biol. J. Linn. Soc. 2007, 90, 211-237. [CrossRef]

37. Steinke, D.; Hanner, R.; Hebert, P.D.N. Rapid high-quality imaging of fishes using a flat-bed scanner. Ichthyol. Res. 2009, 56, 210-211. [CrossRef]

38. Steinke, D.; Hanner, R. The FISH-BOL collaborators' protocol. Mitochondrial DNA 2011, 22 (Suppl. S1), 10-14. [CrossRef]

39. Jacquin, L.; Gauthey, Z.; Roussille, V.; Le Hénaff, M.; Tentelier, C.; Labonne, J. Melanin in a changing world: Brown trout coloration reflects alternative reproductive strategies in variable environments. Behav. Ecol. 2017, 28, 1423-1434. [CrossRef]

40. Selz, O.M.; Thommen, R.; Pierotti, M.E.R.; Anaya-Rojas, J.M.; Seehausen, O. Differences in male coloration are predicted by divergent sexual selection between populations of a cichlid fish. Proc. R. Soc. B Biol. Sci. 2016, 283, 20160172. [CrossRef]

41. Wright, D.S.; Rietveld, E.; Maan, M.E. Developmental effects of environmental light on male nuptial coloration in Lake Victoria cichlid fish. PeerJ 2018, 6, e4209. [CrossRef]

42. Endler, J.A. On the measurement and classification of colour in studies of animal colour patterns. Biol. J. Linn. Soc. 1990, 41, 315-352. [CrossRef]

43. Culumber, Z.W.; Monks, S. Does fin coloration signal social status in a dominance hierarchy of the livebearing fish Xiphophorus variatus? Behav. Process. 2014, 107, 158-162. [CrossRef] [PubMed]

44. Atsumi, K.; Koizumi, I. Web image search revealed large-scale variations in breeding season and nuptial coloration in a mutually ornamented fish, Tribolodon hakonensis. Ecol. Res. 2017, 32, 567-578. [CrossRef]

45. Endler, J.A.; Mielke, P.W., Jr. Comparing entire colour patterns as birds see them. Biol. J. Linn. Soc. 2005, 86, 405-431. [CrossRef]

46. Gerald, M.S.; Bernstein, J.; Hinkson, R.; Fosbury, R.A. Formal method for objective assessment of primate color. Am. J. Primatol. Off. J. Am. Soc. Primatol. 2001, 53, 79-85. [CrossRef]

47. Svensson, P.A.; Nilsson Sköld, H. Skin biopsies as tools to measure fish coloration and colour change. Ski. Biopsy Perspect. Croat. Intech. Open 2011, 299-316. [CrossRef]

48. Fujii, R. The regulation of motile activity in fish chromatophores. Pigment. Cell Res. 2000, 13, 300-319. [CrossRef]

49. Korzan, W.J.; Fernald, R.D. Territorial male color predicts agonistic behavior of conspecifics in a color polymorphic species. Behav. Ecol. 2007, 18, 318-323. [CrossRef]

50. Plenderleith, M.; van Oosterhout, C.; Robinson, R.L.; Turner, G.F. Female preference for conspecific males based on olfactory cues in a Lake Malawi cichlid fish. Biol. Lett. 2005, 1, 411-414. [CrossRef]

51. Yamamoto, T. Sex Differentiation. In Fish Physiology; Hoar, W.S., Randall, D., Eds.; Academic Press: New York, NY, USA, 1976; Volume 3, pp. 117-175.

52. Strüssmann, C.A.; Nakamura, N. Morphology, endocrinology, and environmental modulation of gonadal sex differentiation in teleost fishes. Fish Physiol. Biochem. 2002, 26, 13-29. [CrossRef]

53. Beeching, S.C. Colour pattern and inhibition of aggression in the cichlid fish Astronotus ocellatus. J. Fish Biol. 1995, 47, 50-58. [CrossRef]

54. Fisher, H.S.; Rosenthal, G.G. Male swordtails court with an audience in mind. Biol. Lett. 2007, 3, 5-7. [CrossRef] [PubMed]

55. Leclercq, E.; Taylor, J.F.; Migaud, H. Morphological skin colour changes in teleosts. Fish Fish. 2010, 11, 159-193. [CrossRef]

56. Dickman, M.C.; Schliwa, M.; Barlow, G.W. Melanophore death and disappearance produces color metamorphosis in the polychromatic Midas cichlid (Cichlasoma citrinellum). Cell Tissue Res. 1988, 253, 9-14. [CrossRef] [PubMed]

57. Quigley, I.K.; Turner, J.M.; Nuckels, R.J.; Manuel, J.L.; Budi, E.H.; MacDonald, E.L.; Parichy, D.M. Pigment pattern evolution by differential deployment of neural crest and post-embryonic melanophore lineages in Danio fishes. Development 2004, 131, 6053-6069. [CrossRef]

58. Bagnara, J.T.; Taylor, J.D.; Hadley, M.E. The dermal chromatophore unit. J. Cell Biol. 1968, 38, 67-79. [CrossRef]

59. Takahashi, D. Female mate choice based on male nuptial coloration in pale chub Zacco Platypus. Zool. Sci. 2018, 35, 23-27. [CrossRef] 
60. Ansai, S.; Mochida, K.; Fujimoto, S.; Mokodongan, D.F.; Sumarto, B.K.A.; Masengi, K.W.A.; Hadiaty, R.K.; Nagano, A.J.; Toyoda, A.; Naruse, K.; et al. Genome editing reveals fitness effects of a gene for sexual dichromatism in Sulawesian fishes. Nat. Commun. 2021, 12, 1350. [CrossRef] [PubMed]

61. Grether, G.F. Carotenoid limitation and mate preference evolution: A test of the indicator hypothesis in guppies (Poecilia reticulata). Evol. Int. J. Org. Evol. 2000, 54, 1712-1724. [CrossRef] [PubMed]

62. Godin, J.G.J.; McDonough, H.E. Predator preference for brightly colored males in the Guppy: A viability cost for a sexually selected trait. Behav. Ecol. 2003, 14, 194-200. [CrossRef]

63. Johnson, S.; Candolin, U. Predation cost of a sexual signal in the threespine stickleback. Behav. Ecol. 2017, 28, 1160-1165. [CrossRef]

64. Di Girolamo, N.; Coroneo, M.; Wakefield, D. Epidermal Growth Factor Receptor Signaling Is Partially Responsible for the Increased Matrix Metalloproteinase-1 Expression in Ocular Epithelial Cells after UVB Radiation. Am. J. Pathol. 2005, 167, 489-503. [CrossRef]

65. Kodric-Brown, A. Sexual dichromatism and temporary colour changes in the reproduction of shes. Am. Zool. 1998, 38, 70-81. [CrossRef]

66. Ng'oma, E.; Groth, M.; Ripa, R.; Platzer, M.; Cellerino, A. Transcriptome pro-ling of natural dichromatism in the annualshes Nothobranchius furzeri and Nothobranchius kadleci. BMC Genom. 2014, 15, 754. [CrossRef]

67. Nelissen, M.H.J. Communication. In Cichlid Fishes: Behaviour, Ecology and Evolution; Keenleyside, M.H.A., Ed.; Chapman Hall: London, UK, 1991; pp. 225-240.

68. Hengartner, M.O. The biochemistry of apoptosis. Nature 2000, 407, 770-776. [CrossRef]

69. Sugimoto, M. Morphological color changes in fish: Regulation of pigment cell density and morphology. Microsc. Res. Tech. 2002, 58, 496-503. [CrossRef]

70. Irion, U.; Nüsslein-Volhard, C. The identification of genes involved in the evolution of color patterns in fish. Curr. Opin. Genet. Dev. 2019, 57, 31-38. [CrossRef]

71. Desjardins, J.K.; Fernald, R.D. How do social dominance and social information inuence reproduction and the brain? Integr. Comp. Biol. 2008, 48, 596-603. [CrossRef]

72. Fernald, R.D. Social regulation of reproduction: What changes and why? Horm. Brain Behav. 2009, 1, 683-691.

73. Mikulin, A.E. Functional Significance of Pigments and Pigmentation in Fish Ontogenesis; VNIRO Publishing House: Moscow, Russia, 2000; p. 231. (In Russian) 